\title{
Structural studies of C-reactive Protein
}

\author{
S.M. Collister, W.A. Neale, J.R. Littlejohn, T.J. Greenhough, A.K. Shrive \\ School of Life Sciences, Keele University, U.K. \\ s.collister@keele.ac.uk
}

Human C-reactive protein (CRP) is an innate immune macromolecule of hepatic origin produced in response to inflammatory cytokines. CRP serum concentration is exploited as a clinical biomarker in humans as levels rise rapidly in response to inflammation, infection, or tissue damage [1]. CRP has opsonising abilities and roles in the inflammatory response and activation of complement.

Native human CRP consists of five identical non-covalently bound subunits. The five protomers of CRP are arranged symmetrically around a central pore, consisting of 206 amino acids folded into two antiparallel $\beta$-sheets with a flattened jellyroll topology [2]. Each protomer has a calcium dependent ligand binding site and an effector binding site on the opposite sides of the molecule. The 'recognition' face binds phosphocholine (PC) in a calcium-dependent manner in a ligand binding site located within a hydrophobic pocket. PC is a principal ligand for CRP; widely expressed on the surface of damaged cell membranes and distributed in lipopolysaccharides of bacteria and other microorganisms [1]. PC binding is mediated by a phosphate-calcium interaction. The opposite 'effector' face of CRP accommodates multiple binding sites, for $\mathrm{Clq}$ and immunoglobulin $\mathrm{F} c \gamma$ receptors. The putative $\mathrm{C} 1 \mathrm{q}$ binding site is located at the end of a cleft bordered by the pentraxin helix [3].

Although CRP is remarkably stable under physiological conditions, it has been shown that CRP can dissociate into individual subunits to form monomeric CRP (mCRP). Evidence is increasing that monomeric CRP may have a pro-inflammatory role. We have successfully dissociated CRP, in the presence of urea, into mCRP in-vitro and identified a monomeric CRP with the same reactivity as that seen in patient samples [4]. In addition to the presence of urea, the removal of calcium ions to destabilise the protein is required. Monomeric $\mathrm{CRP}$ has been produced via urea-induced dissociation, optimised at 3M urea over a ten-week period [4]. This CRP form retains its reversible PC-binding ability. Another form of monomeric CRP has been observed in vitro, produced during excessive denaturing conditions, requiring $8 \mathrm{M}$ Urea [5] which does not retain the ability to bind PC. Optimisation of production of these in vitro mCRP forms and crystallisation trials are currently underway.

[1] Sproston, N.R. and Ashworth, J.J. (2018). Role of C-reactive protein at sites of inflammation and infection. Frontiers in Immunology, 9 , 754.

[2] Shrive, A.K., Gheetham, G.M., Holden, D., Myles, D.A., Turnell, W.G., Volanakis, J.E., Pepys, M.B., Bloomer, A.C. and Greenhough, T.J. (1996). Three dimensional structure of human C-reactive protein. Nature Structural Biology, 3(4), 346-354.

[3] Agrawal, A., Shrive, A.K., Greenhough, T.J. and Volanakis, J.E. (2001). Topology and structure of the C1q-binding site on C-reactive protein. The Journal of Immunology, 166(6), 3998-4004.

[4] Williams, R.D., Moran, J.A., Fryer, A.A., Littlejohn, J.R., Williams, H.M., Greenhough, T.J. and Shrive, A.K., 2020. Monomeric C-reactive protein in serum with markedly elevated CRP levels shares common calcium-dependent ligand binding properties with an in vitro dissociated form of C-reactive protein. Frontiers in immunology, 11, p.115.

[5] Potempa LA, Maldonado BA, Laurent P, Zemel ES, Gewurz H. (1983) Antigenic, electrophoretic and binding alterations of human C-reactive protein modified selectively in the absence of calcium. Mol. Immunology, 20:1165-75.

Keywords: C-reactive protein, monomeric C-reactive protein, CRP, C1q 